\title{
A variable neighborhood descent based heuristic to solve the capacitated location-routing problem
}

\author{
M. S. Jabal-Ameli ${ }^{\mathrm{a}}$, M. B. Aryanezhad ${ }^{\mathrm{a}}$ and N. Ghaffari-Nasab ${ }^{\mathrm{a}^{*}}$ \\ ${ }^{a}$ Department of Industrial Engineering, Iran University of Science and Technology, Narmak, Tehran Iran
}

\section{A R T I C L E I N F O}

\section{Article history:}

Received 1 August 2010

Received in revised form

1 September 2010

Accepted 12 September 2010

Available online

12 September 2010

Keywords:

Location-Routing

Logistics

Supply Chain Management

Meta-heuristics

Variable Neighborhood Descent

\section{A B S T R A C T}

\begin{abstract}
Location-routing problem (LRP) is established as a new research area in the context of location analysis. The primary concern of LRP is on locating facilities and routing of vehicles among established facilities and existing demand points. In this work, we address the capacitated LRP which arises in many practical applications within logistics and supply chain management. The objective is to minimize the overall system costs which include the fixed costs of opening depots and using vehicles at each depot site, and the variable costs associated with delivery activities. A novel heuristic is proposed which is based on variable neighborhood descent (VND) algorithm to solve the resulted problem. The computational study indicates that the proposed VND based heuristic is highly competitive with the existing solution algorithms in terms of solution quality.
\end{abstract}

\section{Introduction}

The efficient and effective movement of goods from raw material sites to processing facilities, component fabrication plants, finished goods assembly plants, distribution centers, retailers and customers is critical in today's competitive environment. Approximately $10 \%$ of the gross domestic product of any country is devoted to supply-related activities (Simchi-Levi et al. 2003) and it can easily exceed this value within individual industries. In many real life situations, shipments are made in less-than-truckload (LTL) quantities from a facility to customers along a multiple-stop route. In the case of full truckload quantities, the cost of delivery is independent of the other deliveries made, whereas in the case of LTL quantities, the cost of delivery depends on the other customers on the route and the sequence in which customers are visited.

Location-routing problem is not a single well defined problem like the Weber or the travelling salesman problem. It can be thought of as a combination of sub-problem within location analysis and logistics. However, it is preferred to think of the LRP as an approach for modeling and solving

* Corresponding author. Tel./fax: +98-914-1044339.

E-mail addresses: nghaffari@ind.iust.ac.ir (N. Ghafari-Nasab), 
problems in the location theory. Thus, we use the definition of location-routing introduced by Nagy and Salhi (2007), as "location planning with tour planning aspects taken into account". This definition stems from a hierarchical viewpoint, whereby the aim is to solve a facility location problem (the "master problem"), but in order to achieve this, we also need to solve a vehicle routing problem as the sub-problem. This also implies an integrated solution approach, i.e. an approach that considers both location and routing aspects of a problem but does not address their correlation to the LRP problem. Another important characteristic of above definition is the requirement for the existence of tour planning, i.e. the existence of multiple stops on routes. This specially occurs in situations where customers demands are less than a full truckload, as mentioned earlier. Location-routing problems are closely related to both the classical location-allocation and the vehicle routing problems. In fact, both of the latter problems can be thought of as special cases of the LRP. If we require all customers to be directly linked to the existing depots, the LRP is reduced to a standard location problem. On the other hand, if we fix the depot locations, the LRP becomes a VRP. From a practical viewpoint, locationrouting is a part of distribution management, while theoretically it can usually be modeled as a combinatorial optimization problem. We note that this is an NP-hard problem, since it encompasses two NP-hard problems called facility location and vehicle routing. This paper is organized as follows. Section 2 briefly surveys the literature of location-routing problem. In section 3 a mathematical formulation of the capacitated location-routing problem (CLRP) is introduced. In section 4, the details of the implementation of VND-based heuristic to solve the CLRP is described. Computational results obtained by applying the presented solution scheme on a series of benchmark problem instances, are reported in section 5. Finally, the paper is concluded in Section 6.

\section{Literature review}

During the past three decades, there have been tremendous efforts on integrated location-routing models. Eilon et al. (1971) were among the first to highlight the error introduced by approximating LTL shipments by full truckloads. Integrated location-routing problem is composed of three crucial sub-problems in supply chain design: facility location, customer allocation to facilities and vehicle routing. So far, many different location-routing problems have been presented in the literature, and they tend to be very difficult to solve since they merge two NP-hard problems: facility location and vehicle routing. Laporte (1988) reviewed early work on location-routing problems where he summarized different types of formulations, solution algorithms and computational results of work published prior to 1988. Laprote et al. (1988) examined a class of asymmetrical multi-depot vehicle routing and location-routing problems, under capacity or maximum cost restrictions. By using a suitable graph representation, and then a graph extension, they transformed the problem into equivalent constrained assignment problems. They found optimal solutions by means of a branch and bound tree and solved problems for up to 80 nodes without difficulty.

More recently, Min et al. (1998) developed a hierarchical taxonomy and classification scheme to review the existing location-routing literature. They categorized different works in terms of problem characteristics and solution methodology. One means of classification was the number of layers of facilities. Typically, three-layer problems include flows from plants to distribution centers to customers, while two-layer problems focus on flows from distribution centers to customers. Tuzun and Burke (1999) presented a two-phase tabu search scheme for solving the LRP. They introduced a two-phase approach which offers a computationally efficient strategy that integrates facility location and routing decisions. An extensive computational study shows that their TS algorithm achieves significant improvement over an existing effective LRP heuristic. Nagy and Salhi (2007) conducted a comprehensive survey of location-routing problems. They proposed a classification scheme and looked at a number of problem variants. Both exact and heuristic algorithms were investigated in their work. Prins et al. (2006) proposed a new two-phase meta-heuristic to solve the capacitated LRP. In the first phase which is based on greedy randomized adaptive search procedure (GRASP), a randomized version of Clarke and Wright (1964) algorithm is implemented. This phase is executed 
with a learning process on the choice of depots. The second phase produces new solutions using a post-optimization and path re-linking. Barreto et al. (2007) considered a discrete LRP with two levels: a set of potential capacitated distribution centers (DC) and a set of ordered customers. They used a number of hierarchical and non-hierarchical clustering techniques along with several proximity functions and integrated them in a sequential heuristic algorithm for the above mentioned LRP model. Prins et al. (2007) presented a cooperative heuristic to solve the capacitated locationrouting problem (CLRP). Their heuristic is based on the principle of alternating between a depot location phase and a routing phase, exchanging information on the most promising edges. Marinakis and Marinaki (2008) considered a large-scale real-world problem of location-routing within food industry in Greece and proposed a new formulation of the LRP based on bi-level programming to solve their proposed model. In any LRP problem, decisions are made at a strategic level and at an operational level. Therefore, they formulated the problem in such a way that in the first level, the decisions of the strategic level are made, namely, the top manager finds the optimal location of the facilities; while in the second level, the operational level decisions are made, namely, the operational manager finds the optimal routing of vehicles. Lin and Lei (2009) formulated and analyzed a strategic design model for three-echelon distribution systems in which the routing problem is considered in two levels. In their analysis the clients are divided into two categories: big clients and normal clients, where the big ones have larger demands. To solve such a problem, they developed a hybrid genetic algorithm embedded with a routing heuristic and showed that their algorithm efficiently finds nearoptimal solutions. Schwardt and Fischer (2009) addressed the single-depot location-routing problem and proposed a neural network approach based on a self-organizing map to solve the resulted problem. They compared their results with some well-known heuristics and reported that the selforganizing map approach competes well with them. Hassan-Pour et al. (2009) presented a novel biobjective mathematical programming model for a stochastic location-routing problem (SLRP) in which the first objective is to minimize costs associated with establishing the facilities as well as the transportation cost, and the second objective maximizes the probability of delivery to customers. In their proposed model, new aspects of a LRP such as stochastic availability of facilities and routes are considered. The proposed model is solved in two stages: (i) solving the facility location problem (FLP) by a mathematical algorithm and (ii) solving the multi-objective multi-depot vehicle routing problem (MO-MDVRP) by a simulated annealing (SA) algorithm hybridized by genetic operators, namely mutation and crossover. Yu et al. (2010) proposed an efficient simulated annealing (SA) based heuristic to solve the LRP. They tested their SA heuristic on three sets of well-known benchmark instances and compared the results with other heuristics in the literature. Their proposed solution algorithm provides best known solutions for most of the benchmark instances in the three above mentioned problem sets but the time required to reach such solutions is significantly larger than that of the similar solution algorithms. Duhamel et al. (2010) considered the capacitated location-routing problem and proposed solution method based on greedy randomized adaptive search procedure (GRASP), hybridized with an evolutionary local search (ELS). The proposed framework is benchmarked on classical LRP instances and numerical experiments indicate that the approach outperforms most of the previously published methods and provides numerous new best solutions.

\section{The mathematical programming formulation}

This section presents an integer programming formulations for the capacitated location-routing problem (CLRP). In this model which was proposed by Prins et al. (2007), the assumption of singlesourcing holds. In other words, it is assumed that the customers acquire their needed demand from a single supplier. CLRP imposes constraints on both the vehicle capacities and the depot capacities. The following notations are used to represent the mathematical programming formulation. 
Sets and parameters:

$J \quad$ Set of customers indexed by $j$

I Set of candidate depot sites indexed by $i$

$K \quad$ Set of vehicles indexed by $k$

$V \quad$ Set of all points; $V=J \cup I$

$E \quad$ Set of $\operatorname{arcs}(i, j)$ connecting every pair of nodes $i, j \in V$

$q_{j} \quad$ Demand of customer $j$

$O_{i} \quad$ Fixed cost of opening a depot at candidate site $i$

$F_{i} \quad$ Fixed cost of employing a vehicle at candidate site $i$

$P_{i} \quad$ Capacity of depot to be located at candidate site $i$

$c_{i j} \quad$ Cost of traveling associated with arc $(i, j) \in E$

$Q \quad$ Capacity of vehicles; here it is assumed that all vehicles are homogeneous

Decision variables:

$$
\begin{aligned}
& Z_{i}= \begin{cases}1 & \text { if we open a depot at candidate site } i \\
0 & \text { if not }\end{cases} \\
& Y_{i j}= \begin{cases}1 & \text { if demands at customer } j \text { are served by the depot at candidate site } i \\
0 & \text { if not }\end{cases} \\
& X_{i j}= \begin{cases}1 & \text { if vehicle } k \text { goes directly from node } i \text { to node } j \\
0 & \text { if not }\end{cases}
\end{aligned}
$$

The mathematical programming formulation for the capacitated location-routing problem (CLRP) is as follows,

$\min \sum_{i \in I} O_{i} Z_{i}+\sum_{i \in I} \sum_{j \in J \in \in K K} F_{i} X_{i j k}+\sum_{i \in V} \sum_{j \in V} \sum_{k \in K} c_{i j} X_{i j k}$

subject to

$$
\begin{array}{ll}
\sum_{i \in U} \sum_{k \in K} X_{i j k}=1 & \forall j \in J \\
\sum_{i \in} \sum_{j \in J} q_{j} X_{i j k} \leq Q & \forall k \in K \\
\sum_{j \in J} q_{j} Y_{i j} \leq P_{i} Z_{i} & \forall i \in I
\end{array}
$$




$$
\begin{array}{ll}
\sum_{i \in S} \sum_{j \in S} X_{i j k} \leq|S|-1 & \forall S \subseteq J ; \forall k \in K \\
\sum_{j \in V} X_{i j k}-\sum_{j \in V} X_{j i k}=0 & \forall j \in V ; \forall k \in K \\
\sum_{i \in I} \sum_{j \in J} X_{i j k} \leq 1 & \forall k \in K \\
\sum_{m \in V} X_{i m k}+\sum_{h \in V} X_{j h k} \leq 1+Y_{i j} & \forall i \in I ; \forall j \in J ; \forall k \in K \\
Z_{i} \in\{0,1\} & \forall i \in I \\
Y_{i j} \in\{0,1\} & \forall i \in I ; \forall j \in J \\
X_{i j k} \in\{0,1\} & \forall i \in V ; \forall j \in V ; \forall k \in K
\end{array}
$$

The three terms in the objective function (1) represent the sum of the fixed depot location cost and routing costs including the fixed costs of employing vehicles and the travel costs, respectively. Constraints (2) ensure that each customer belongs to one and exactly one route, and that each customer has only one predecessor in the route. Inequalities (3) and (4) impose constraints on the capacity of the routes and depots, respectively. Constraints (5) are the standard sub-tour elimination constraints which indicate that for any subset $S$ of the set of customers $J$ and for any route $k$, the number of arcs belonging to route $k$ that connect the members of $S$, must not exceed the cardinality of $S$ minus one. Constraints (6) and (7) guarantee the continuity of each route, and that each route terminates at the depot where the route starts. Constraints (8) ensure that a customer must be allocated to a depot if there is a route connecting them. Finally, (9), (10), and (11) are integrality constraints.

\section{Variable neighborhood descent heuristic for the CLRP}

Variable neighborhood descent (VND) is the deterministic version of the variable neighborhood search (VNS) meta-heuristic proposed by Mladenovic and Hansen (1997). The basic idea of VND (or VNS, in general) is to successively explore a set of predefined neighborhood structures to provide a better solution. It systematically explores a set of neighborhoods to get different local optima and to escape from local optima. VND exploits the following two important facts: 1) using various neighborhoods in local search may generate different local optima, and 2) the global optimum is a local optimum for a given neighborhood (Talbi 2009). In the following sections we first discuss the solution representation method employed in this study and then describe the proposed solution procedure which is composed of two phases: the initial solution generation phase and solution improvement phase.

\subsection{Solution Representation}

In this research we propose a new method to represent the solutions to the LRP. The new representation uses two dimensional arrays (matrices), in which each row represents a route. The first entry of each row shows the depot from which the corresponding route originates. The next entries at each row show the sequence according to which the customers must be visited. Fig. 1 shows a solution to a typical LRP instance with 20 customers and 6 candidate sites for depot locations. As it is depicted, in this solution three depots of 22, 23 and 25, out of six, are open. The deliveries are made though five established routes: two routes are originated from depot 22, two routes from depot 23, and one route from depot 25 . The corresponding matrix representation is also depicted in Fig. 1. 


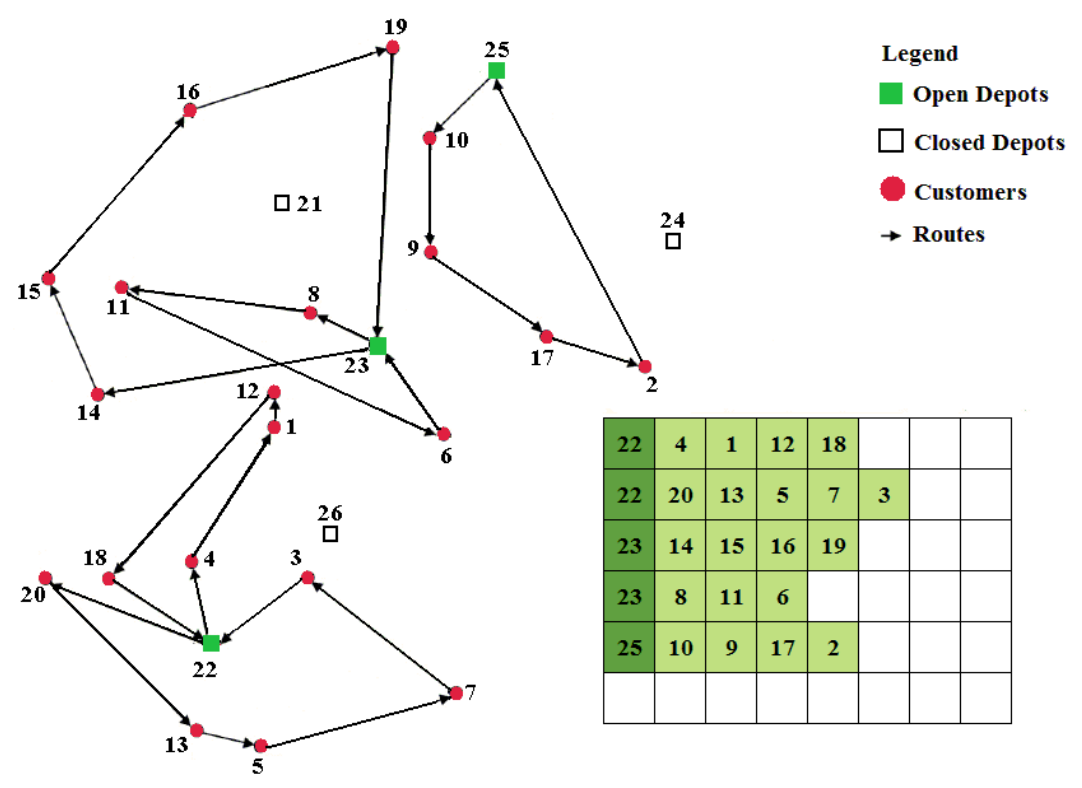

Fig. 1. A solution for a typical LRP and its corresponding matrix representation

As we can observe, the matrix representation comprises five rows where each row is associated with an established route. Consider the last route originated from depot 25 of the matrix representation. The customers 10, 9, 17, and 2 are covered by this route and they are visited in the same order as shown in the last row of the matrix.

\subsection{Phase I: Initial solution generation}

The quality of the initial solution plays an important role in the quality of the final solutions obtained by the VND based heuristic. In this study we propose a two-stage algorithm to generate relatively high quality initial solutions. In the first stage, the open depots are located and customers are allocated to them (location-allocation), while in the second stage, the delivery routes are established using the Clarke and Wright's savings algorithm (Clarke \& Wright, 1964), which is one of the most known heuristics for the vehicle routing problem in the literature (see appendix for detailed description of Clarke and Wright's savings algorithm). Steps of the proposed algorithm for generating the initial solution are shown in Fig. 2.

Initialization. For every candidate depot site $j$, define the set $S_{j}$ to be the set of all customers to whom the site $j$ is the nearest depot site; for every $j \in J$.

Location-Allocation. Open the depot $j$ if $S_{j} \neq \varnothing$, and allocate all the customers in the $S_{j}$ to the depot $j$; for every $j \in J$.

Routing. For every open depot, use the Clark-Wright algorithm to solve the corresponding vehicle routing problem.

Fig. 2. Initial solution generation

\subsection{Phase II: Improvement of the initial solution}

Once the initial solution is generated, we can improve its quality using the VND algorithm. As mentioned earlier in this section, VND uses successive neighborhoods in descent to a local optimum. The outline of the VND algorithm can be simply described as follows. First, one needs to define a set of neighborhood structures $N_{l}\left(l=1, \ldots, l_{\max }\right)$. Let $N_{1}$ be the first neighborhood structure to be used and $x$ be the initial solution. At each stage of the search procedure, if it is not possible to find an 
improvement of the solution $x$ in its current neighborhood $N_{l}(x)$, the neighborhood structure is changed from $N_{l}$ to $N_{l+1}$. On the other hand, if an improvement of the current solution $x$ is found, the algorithm restarts the search with the first neighborhood structure $N_{1}(x)$. This strategy will be effective if the different neighborhood structures used are complementary in the sense that a local optimum for a neighborhood structure $N_{i}$ will not be a local optimum in the neighborhood structure $N_{j}$. The entire scheme of the VND algorithm is shown in Fig. 3.

Initialization. Select the set of neighborhood structures $N_{l}$, for $l=1, \ldots$ , $l_{\max }$, used in the descent and find an initial solution $x$ (or apply the rules to a given $x$ );

$\underline{\text { Repeat }}$ the following sequence until no improvement is obtained:

(1) Set $l \leftarrow 1$;

(2) $\underline{\text { Repeat }}$ the following steps until $l=l_{\text {max }}$ :

(a) Exploration of neighborhood. Find the best neighbor $x^{\prime}$ of $x$ $\left(x^{\prime} \in N_{l}(x)\right)$

(b) Move or not. If the solution $x^{\prime}$ thus obtained is better than $x$, set

$x \leftarrow x^{\prime}$ and $l \leftarrow 1$; otherwise, set $l \leftarrow l+1$;

Fig. 3. Steps of the VND algorithm

\subsubsection{Defined neighborhoods}

The efficiency of any meta-heuristic algorithm which uses neighborhood structures to search the solution space severely depends on how the neighborhood structures used in the algorithm are defined. The number of neighborhood structure and the order according to which these structures are used in the local search procedure affect both the quality of the solutions and the amount of time needed to reach such solutions. We propose the following seven neighborhood structures in our study to search the solution space in the VND algorithm. The first four neighborhood structures affect the location-allocation related part to the solutions while the last three neighborhood structures address the routing related part of the solutions.

Neighborhood structure 1 (Opening a closed depot). This move randomly selects a closed depot, if there is any, and opens it. Then some customers from other open depots are allocated to the newly opened depot according to their proximity. Fig. 4 illustrates this move applied to a typical solution in the matrix representation format.

Neighborhood structure 2 (Closing an open depot). This move randomly selects an open depot and closes it. Then the customers allocated to the newly closed depot are re-allocated to other open depots according to their proximity. Fig. 5 illustrates this move.

Neighborhood structure 3 (Customer reallocation move). In this move one customer from a randomly selected route is chosen and reallocated to another randomly selected route. Fig. 6 illustrates the customer reallocation move.

Neighborhood structure 4 (Customer exchange move). In this move two customers from two different randomly selected routes are chosen and their positions are exchanged in the corresponding routes. Fig. 7 illustrates the customer exchange move. 


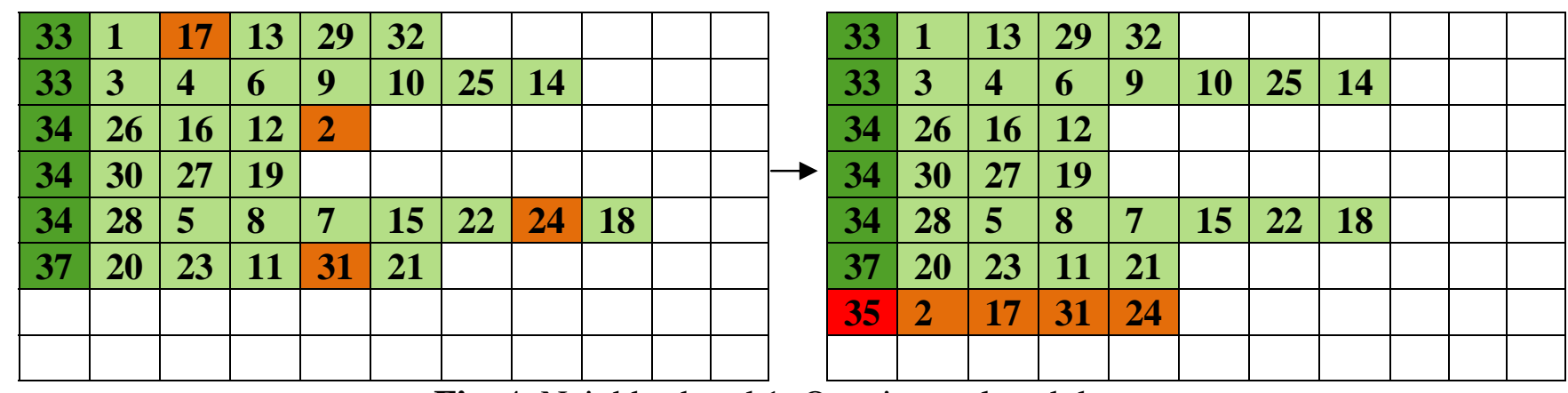

Fig. 4. Neighborhood 1: Opening a closed depot

\begin{tabular}{|l|l|l|l|l|l|l|l|l|l|l}
33 & 1 & 17 & 13 & 29 & 32 & & & & & \\
\hline 3 & 3 & 4 & 6 & 9 & 10 & 25 & 14 & & & \\
\hline 34 & 26 & 16 & 12 & 2 & & & & & & \\
\hline 34 & 30 & 27 & 19 & & & & & & & \\
\hline 34 & 28 & 5 & 8 & 7 & 15 & 22 & 24 & 18 & & \\
\hline 37 & 20 & 23 & 11 & 31 & 21 & & & & & \\
\hline & & & & & & & & & &
\end{tabular}$\rightarrow$\begin{tabular}{|l|l|l|l|l|l|l|l|l|l|l|l|}
34 & 26 & 16 & 12 & 2 & 3 & 4 & & & & \\
\hline 34 & 30 & 27 & 19 & 6 & 9 & 1 & 17 & 13 & & \\
\hline 34 & 28 & 5 & 8 & 7 & 15 & 22 & 24 & 18 & 29 & \\
\hline 37 & 20 & 23 & 11 & 31 & 21 & 10 & 25 & 14 & 32 & \\
\hline & & & & & & & & & & \\
\hline & & & & & & & & & & \\
\hline & & & & & & & & & & \\
\hline
\end{tabular}

Fig. 5. Neighborhood 2: Closing an open depot

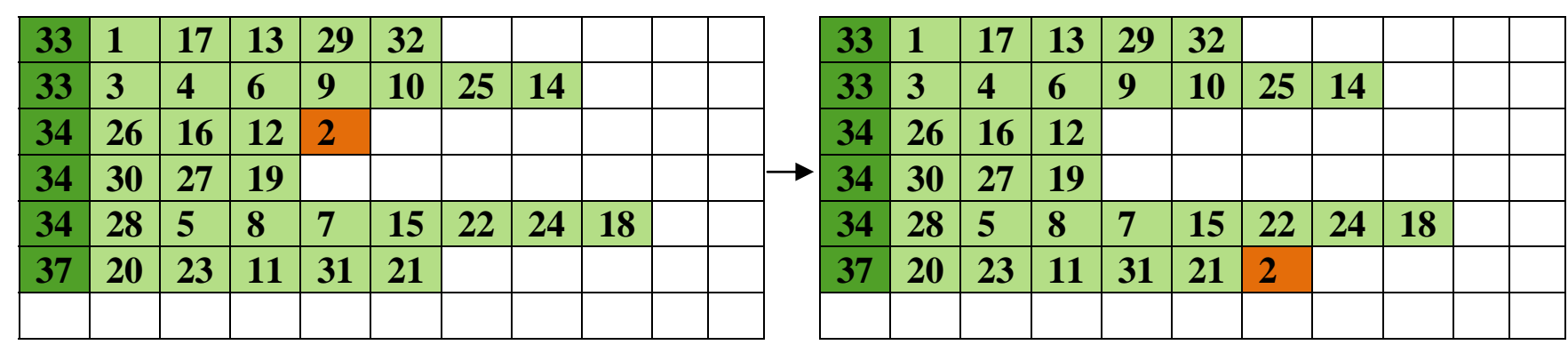

Fig. 6. Neighborhood 3: Customer reallocation move

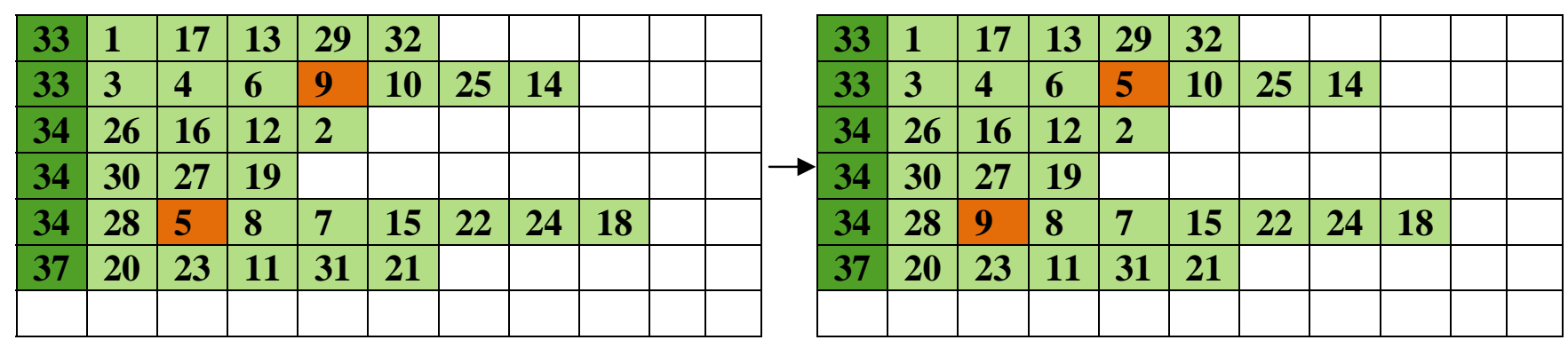

Fig. 7. Neighborhood 4: Customer exchange move

Neighborhood structure 5 (2-opt move). 2-opt move which is commonly employed to solve the vehicle routing related problems selects a route and performs 2-opt move on it as depicted in Fig. 8. In terms of matrix representation, we can describe this move as follows. A sub-sequence of customers is selected and the order of the customers is reversed in that sub-sequence. Fig. 9 illustrates the 2-opt move in the matrix representation format. 
Neighborhood structure 6 (3-opt move). This move is commonly used in vehicle routing related problems by selecting a route and performs 3-opt move on it as illustrated in Fig. 10. In terms of matrix representation, we can describe this move as follows. Sub-sequence of customers is selected and they are shifted backward in the sequence of customers associated with that route. Fig. 11 illustrates the 3-opt move in the matrix representation format.

Neighborhood structure 7 (Route split move). In this move, one route (master route) is randomly selected and it is divided into two sub-routes. These newly generated sub-routes are both assigned to the same depot as the master route. Fig. 12 demonstrates the details of the step.

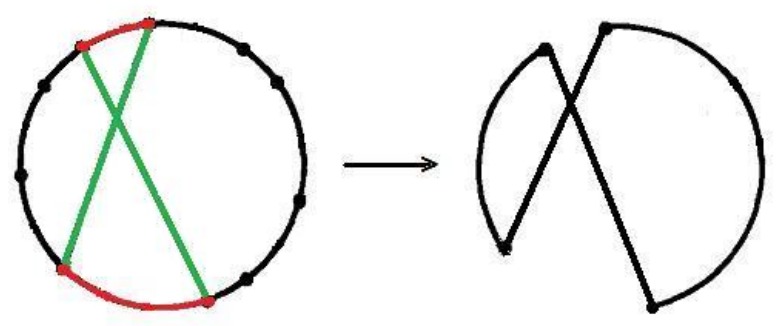

Fig. 8. Neighborhood 5: 2-opt move

\begin{tabular}{|l|l|l|l|l|l|l|l|l|l|l|}
\hline 33 & 1 & 17 & 13 & 29 & 32 & & & & & \\
\hline 33 & 3 & 4 & 6 & 9 & 10 & 25 & 14 & & & \\
\hline 34 & 26 & 16 & 12 & 2 & & & & & & \\
\hline 34 & 30 & 27 & 19 & & & & & & & \\
\hline 34 & 28 & 5 & 8 & 7 & 15 & 22 & 24 & 18 & & \\
\hline 37 & 20 & 23 & 11 & 31 & 21 & & & & & \\
\hline & & & & & & & & & & \\
\hline
\end{tabular}

\begin{tabular}{|l|l|l|l|l|l|l|l|l|l|l|}
\hline 33 & 1 & 17 & 13 & 29 & 32 & & & & & \\
\hline 33 & 3 & 4 & 6 & 9 & 10 & 25 & 14 & & & \\
\hline 34 & 26 & 16 & 12 & 2 & & & & & & \\
\hline 34 & 30 & 27 & 19 & & & & & & & \\
\hline 34 & 28 & 5 & 24 & 22 & 15 & 7 & 8 & 18 & & \\
\hline 37 & 20 & 23 & 11 & 31 & 21 & & & & & \\
\hline & & & & & & & & & & \\
\hline
\end{tabular}

Fig. 9. Graphical illustration of 2-opt move (from left to right)

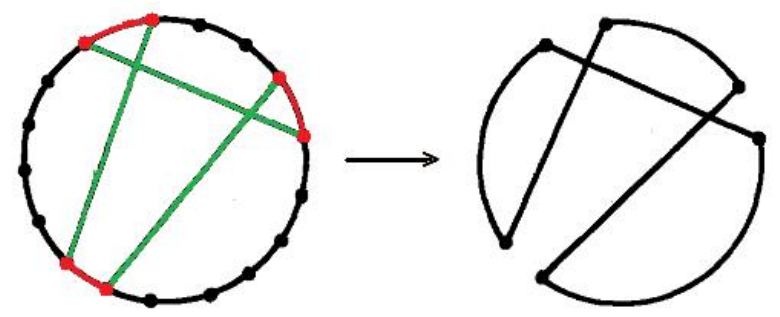

Fig. 10. Graphical illustration of 3-opt move

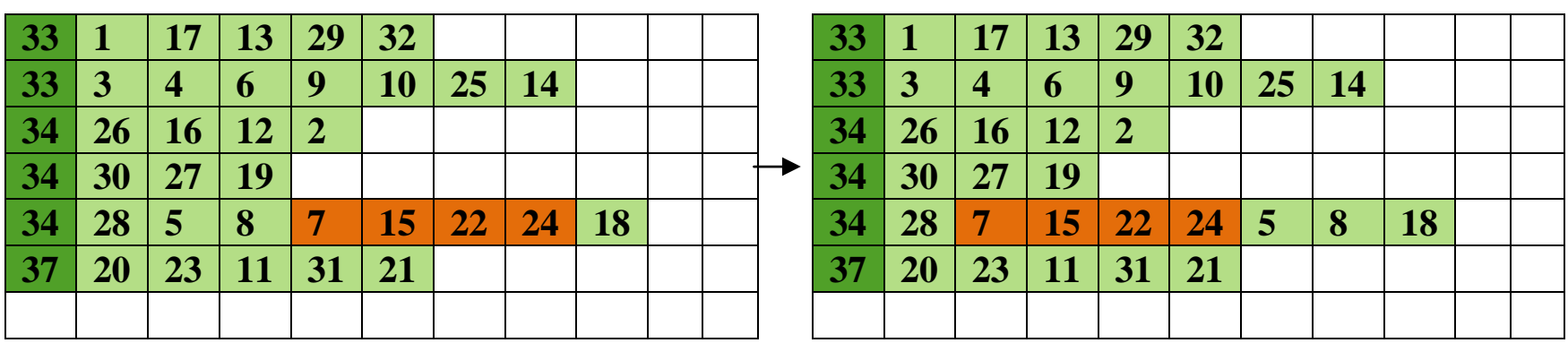

Fig. 11. Neighborhood 6: 3-opt move (from left to right) 


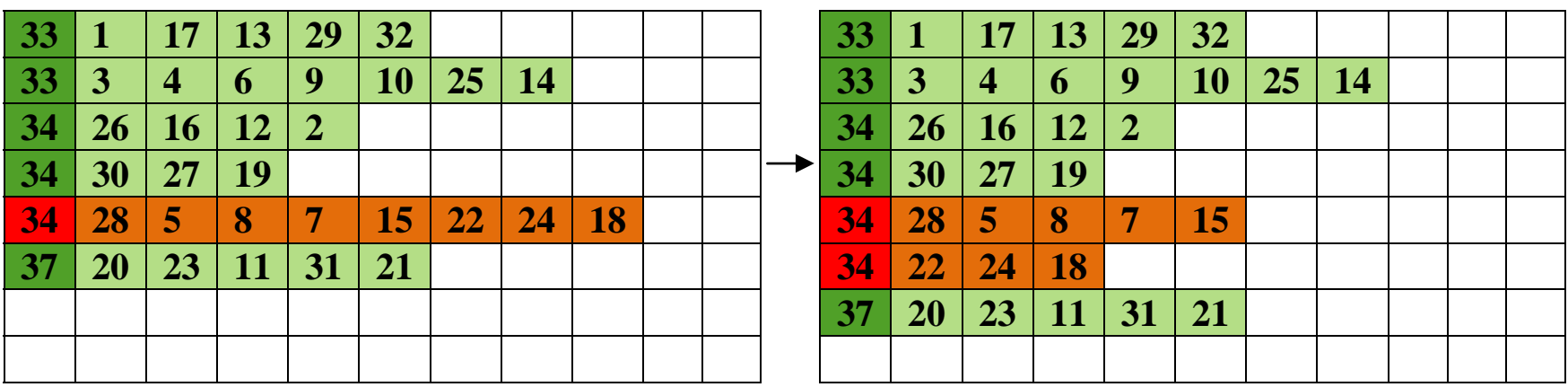

Fig. 12. Neighborhood 7: Route split move

\section{Computational study}

In this section, we evaluate the performance of VND based heuristic to solve the CLRP. The proposed solution algorithm is coded in MATLAB R2007b and it was run on a PC with an Intel Core 2 Duo CPU $(2.0 \mathrm{GHz})$ and $2 \mathrm{~GB}$ memory. In order to verify the proposed approach, two well-known LRP benchmark problem sets are selected and results obtained by applying the VND on these problem sets are compared to the best known solutions provided by some efficient LRP heuristics available in the literature.

\subsection{The benchmark problem sets}

In this research, we have evaluated the performance of the proposed solution procedure using two important benchmark problem sets on the literature. The first set of benchmark problems, designed by Tuzun and Burke, comprises 36 instances in which the routes are assumed to be capacitated but the depots are assumed to be uncapacitated. This problem set was used by the authors to evaluate the efficiency of their tabu search heuristic (Tuzun \& Burke, 1999). The numbers of clients in their benchmark instances are set to be 100,150 or 200 . The number of depots is either 10 or 20 . The vehicle capacity is set to be 150 for all instances, and the customer demands are assumed to be uniformly distributed in the interval of $[1,20]$. In this data set, the distances and the travel costs are not rounded. The second set of benchmark problems consists of 19 instances gathered by Barreto (2004). These instances are either gathered from the literature or they are obtained by adding candidate sites for depot locations to existing classical VRP instances. All routes in this problem set are capacitated, and except for a few instances, the depots are also capacitated. The fixed costs associated with employing vehicles are set to be zero in this problem set and the traveling costs are not rounded (Yu et al. 2010).

\subsection{Parameter setting}

Unlike many other meta-heuristics, the VND and other extensions of the VNS are simple and require few, and sometimes no parameters in their design. Therefore, VND provides very good solutions often in simpler ways than other meta-heuristic methods (Hansen \& Mladenovic, 2003). In addition, VND gives some insight for such a performance, which, in turn, can lead to more efficient and sophisticated implementations. In our algorithm, there are only two parameters, which are the unit penalty costs associated with the violation of depot and vehicle capacities $\left(P_{\text {depot }}, P_{\text {vehicle }}\right)$. These parameters have nothing to do with the VND and they must be used in any solution algorithm regardless of their types. In this study, we have set this penalty costs to be 400 for both depot and vehicle capacity. Note that the penalty costs are set to zero in cases where the depots or vehicles are assumed to be uncapacitated.

\subsection{Evaluation of the results}

In summary, we solved 55 LRP benchmark instances taken from two well-known LRP benchmark problem sets existing in the literature to test the performance of the proposed VND heuristic. In order 
to evaluate the efficiency of our heuristic, we have compared the results obtained by VND algorithm by those obtained by the three similar works in the literature, simulated annealing (SA) based heuristic, proposed by $\mathrm{Yu}$ et al. (2010), granular randomized adaptive search procedure (GRASP) based heuristic developed by Prins et al. (2006), and Lagrangian relaxation granular tabu search (LRGTS) heuristic by Prins et al. (2007). Note that, the three above-mentioned heuristics are among the most efficient LRP heuristics in the literature ( $\mathrm{Yu}$ et al., 2010). The results of implementation of VND on the instances of the first and the second problem sets are reported in Tables 1 and 2, respectively.

Table 1

Computational results for the first problem set

\begin{tabular}{|c|c|c|c|c|c|c|c|c|c|}
\hline & & & VND & & & & STS & & $\mathrm{ASP}$ \\
\hline ID & $\mathrm{m}$ & $\mathrm{n}$ & $\mathrm{OFV}$ & OFV & \%Gap & OFV & \%Gap & OFV & \%Gap \\
\hline $\mathrm{T} 1$ & 10 & 100 & 1580 & 1477 & 6.97 & 1491 & 5.97 & 1525 & 3.61 \\
\hline T2 & 20 & 100 & 1556 & 1471 & 5.78 & 1472 & 5.71 & 1527 & 1.90 \\
\hline T3 & 10 & 100 & 1461 & 1409 & 3.69 & 1412 & 3.47 & 1424 & 2.60 \\
\hline T4 & 20 & 100 & 1560 & 1432 & 8.94 & 1443 & 8.11 & 1482 & 5.26 \\
\hline T5 & 10 & 100 & 1242 & 1177 & 5.52 & 1188 & 4.55 & 1200 & 3.50 \\
\hline T6 & 20 & 100 & 1137 & 1110 & 2.43 & 1116 & 1.88 & 1124 & 1.16 \\
\hline T7 & 10 & 100 & 830 & 792 & 4.80 & 813 & 2.09 & 814 & 1.97 \\
\hline T8 & 20 & 100 & 743 & 732 & 1.50 & 743 & 0.00 & 748 & -0.67 \\
\hline T9 & 10 & 100 & 1363 & 1238 & 10.10 & 1268 & 7.49 & 1273 & 7.07 \\
\hline T10 & 20 & 100 & 1340 & 1247 & 7.46 & 1256 & 6.69 & 1273 & 5.26 \\
\hline T11 & 10 & 100 & 926 & 902 & 2.66 & 913 & 1.42 & 912 & 1.54 \\
\hline T12 & 20 & 100 & 1066 & 1024 & 4.10 & 1026 & 3.90 & 1023 & 4.20 \\
\hline T13 & 10 & 150 & 2060 & 1954 & 5.42 & 1946 & 5.86 & 2007 & 2.64 \\
\hline T14 & 20 & 150 & 1952 & 1899 & 2.79 & 1876 & 4.05 & 1889 & 3.34 \\
\hline T15 & 10 & 150 & 2088 & 2057 & 1.51 & 2011 & 3.83 & 2034 & 2.65 \\
\hline T16 & 20 & 150 & 1915 & 1801 & 6.33 & 1820 & 5.22 & 1856 & 3.18 \\
\hline T17 & 10 & 150 & 1593 & 1453 & 9.64 & 1449 & 9.94 & 1508 & 5.64 \\
\hline T18 & 20 & 150 & 1480 & 1456 & 1.65 & 1493 & -0.87 & 1457 & 1.58 \\
\hline T19 & 10 & 150 & 1233 & 1206 & 2.24 & 1211 & 1.82 & 1240 & -0.56 \\
\hline T20 & 20 & 150 & 949 & 935 & 1.50 & 937 & 1.28 & 941 & 0.85 \\
\hline T21 & 10 & 150 & 1820 & 1720 & 5.81 & 1729 & 5.26 & 1737 & 4.78 \\
\hline T22 & 20 & 150 & 1489 & 1416 & 5.16 & 1425 & 4.49 & 1426 & 4.42 \\
\hline T23 & 10 & 150 & 1262 & 1217 & 3.70 & 1216 & 3.78 & 1224 & 3.10 \\
\hline T24 & 20 & 150 & 1231 & 1159 & 6.21 & 1162 & 5.94 & 1231 & 0.00 \\
\hline T25 & 10 & 200 & 2401 & 2324 & 3.31 & 2297 & 4.53 & 2384 & 0.71 \\
\hline T26 & 20 & 200 & 2272 & 2258 & 0.62 & 2208 & 2.90 & 2288 & -0.70 \\
\hline T27 & 10 & 200 & 2299 & 2260 & 1.73 & 2261 & 1.68 & 2273 & 1.14 \\
\hline T28 & 20 & 200 & 2409 & 2327 & 3.52 & 2260 & 6.59 & 2345 & 2.73 \\
\hline T29 & 10 & 200 & 2164 & 2113 & 2.41 & 2121 & 2.03 & 2137 & 1.26 \\
\hline T30 & 20 & 200 & 1798 & 1723 & 4.35 & 1738 & 3.45 & 1807 & -0.50 \\
\hline T31 & 10 & 200 & 1551 & 1469 & 5.58 & 1489 & 4.16 & 1497 & 3.61 \\
\hline T32 & 20 & 200 & 1120 & 1089 & 2.85 & 1091 & 2.66 & 1096 & 2.19 \\
\hline T33 & 10 & 200 & 2119 & 1994 & 6.27 & 1984 & 6.80 & 2045 & 3.62 \\
\hline T34 & 20 & 200 & 2014 & 1932 & 4.24 & 1986 & 1.41 & 2091 & -3.68 \\
\hline T35 & 10 & 200 & 1882 & 1779 & 5.79 & 1787 & 5.32 & 1789 & 5.20 \\
\hline T36 & 20 & 200 & 1418 & 1396 & 1.58 & 1401 & 1.21 & 1409 & 0.64 \\
\hline \multicolumn{3}{|c|}{ Average } & & & 4.39 & & 4.02 & & 2.37 \\
\hline
\end{tabular}


In Tables 1 and 2, the first column represents the problem identification code developed and used by $\mathrm{Yu}$ et al. (2010). The second and the third columns denote the number of candidate sites for depot locations and the number of customers, respectively. The next column contains the objective function values obtained by VND heuristic for each problem instance. Columns five and six represent the objective function values provided by SA algorithm and the relative gap (in percentage) between the objective function values of the SA heuristic and those of the VND heuristic, respectively. The same comparative results for the LRGTS heuristic are reported in columns 7 and 8. Columns 9 and 10 include the objective function values and the relative gap for the GRASP heuristic. According to the results reported in Tables 1 and 2, the VND algorithm provides high quality solutions with the objective function values not more than $5 \%$ (in average) of the objective values of the solutions obtained by the other mentioned heuristics (SA, LRGTS, and GRASP).

Table 2

Computational results for the second problem set

\begin{tabular}{llllllllll}
\hline & & & VND & \multicolumn{2}{c}{ SA } & \multicolumn{2}{c}{ LRGTS } & \multicolumn{2}{c}{ GRASP } \\
\hline ID & $\mathrm{m}$ & $\mathrm{n}$ & OFV & OFV & \%Gap & OFV & \%Gap & OFV & \%Gap \\
\hline B1 & 5 & 21 & 451 & 425 & 6.11 & 425 & 6.12 & 425 & 6.12 \\
B2 & 5 & 22 & 591 & 585 & 1.02 & 587 & 0.68 & 585 & 1.03 \\
B3 & 5 & 29 & 512 & 512 & 0.00 & 512 & 0.00 & 515 & -0.58 \\
B4 & 5 & 32 & 583 & 562 & 3.73 & 587 & -0.68 & 572 & 1.92 \\
B5 & 5 & 32 & 522 & 504 & 3.57 & 505 & 3.37 & 504 & 3.57 \\
B6 & 5 & 36 & 492 & 461 & 6.72 & 477 & 3.14 & 460 & 6.96 \\
B7 & 5 & 50 & 601 & 566 & 6.18 & 586 & 2.56 & 599 & 0.33 \\
B8 & 10 & 75 & 898 & 848 & 5.89 & 864 & 3.94 & 862 & 4.18 \\
B9 & 10 & 100 & 918 & 838 & 9.54 & 843 & 8.90 & 862 & 6.50 \\
B10 & 2 & 12 & 204 & 204 & 0.00 & - & - & - & - \\
B11 & 15 & 55 & 1144 & 1113 & 2.78 & - & - & - & - \\
B12 & 7 & 85 & 1665 & 1623 & 2.58 & - & - & - & - \\
B13 & 4 & 318 & 602315 & 563493 & 6.88 & - & - & - & - \\
B14 & 4 & 318 & 738915 & 684164 & 8.00 & - & - & - & - \\
B15 & 5 & 27 & 3068 & 3062 & 0.19 & 3065 & 0.10 & 3062 & 0.20 \\
B16 & 8 & 134 & 6292 & 5709 & 10.21 & 5809 & 8.31 & 5965 & 5.48 \\
B17 & 8 & 88 & 383 & 356 & 7.58 & 369 & 3.79 & 357 & 7.28 \\
B18 & 10 & 150 & 47662 & 45109 & 5.65 & 44386 & 7.38 & 44625 & 6.81 \\
B19 & 14 & 117 & 13451 & 12435 & 8.17 & - & - & - & - \\
\hline Average & & & & 4.98 & & 3.66 & & 3.82 \\
\hline
\end{tabular}

\section{Conclusion}

In this paper, we have developed a variable neighborhood descent based heuristic to solve the capacitated location-routing problem, which is a practical problem arising in logistics and supply chain management. The proposed solution algorithm consists of two phases where the first phase generates an initial solution and the second phase improves the quality of the initial solution generated in the first phase. The proposed solution procedure has been tested on two sets of benchmark problem instances and the results have been compared with those of the three efficient heuristics in the literature. Numerical results indicate that the proposed method of this paper is highly competitive with other solution schemes existing in the literature and provides solutions with relatively good quality. An interesting line for future research is to hybridize the VND algorithm with other meta-heuristic algorithms like simulated annealing or tabu search, so that a more efficient solution procedure be developed. Another promising area for further research is the application of VND algorithm to other combinatorial optimization problems. 


\section{Appendix}

\section{Clarke and Wright's savings algorithm (Larson and Odoni, 1981)}

Consider a depot $D$ and $n$ demand points. Suppose that initially the solution to the VRP consists of using $n$ vehicles and dispatching one vehicle to each one of the $n$ demand points. The total tour length of this solution is, obviously $2 \sum_{i=1}^{n} d(D, i)$. Consider a single vehicle to serve two points, say $i$ and $j$, on a single trip, the total distance traveled is reduced by the following,

$$
s(i, j)=2 d(D, i)+2 d(D, j)-[d(D, i)+d(i, j)+d(D, j)]=d(D, i)+d(i, j)-d(D, j)
$$

The quantity $s(i, j)$ is known as the "savings" resulted from combining points $i$ and $j$ into a single tour. The larger $s(i, j)$ is, the more desirable it becomes to combine $i$ and $j$ in a single tour. However, $i$ and $j$ cannot be combined the resulted tour violates one or more of the constraints of the VRP. The algorithm can now be described as follows.

STEP1 Calculate the savings $s(i, j)=d(D, i)+d(D, j)-d(i, j)$ for every pair $(i, j)$ of demand points

STEP2 Rank the savings $s(i, j)$ and list them in descending order of magnitude called savings list, process the savings list beginning with the top most entry in the list (the largest $s(i, j)$ )

STEP3 For the savings $s(i, j)$ under consideration, include link $(i, j)$ in a route if no route constraints are violated through the inclusion of $(i, j)$ in a route, and if one of the following cases hold

a. neither $i$ nor $j$ has already been assigned to a route, in which case a new route is initiated including both $i$ and $j$.

b. exactly one of the two points ( $i$ or $j$ ) has already been included in an existing route and that point is not interior to that route (a point is interior to a route if it is not adjacent to the depot $D$ in the order of traversal of points), in which case the link $(i, j)$ is added to that same route.

c. both $i$ and $j$ have already been included in two different existing routes and neither point is interior to its route, in which case the two routes are merged.

STEP4 If the savings list $s(i, j)$ has not been exhausted, return to Step 3, process the next entry in the list; otherwise, stop; the solution to the VRP consists of the routes created during Step 3. (Any points that have not been assigned to a route during Step 3 must each be served by a vehicle route that begins at the depot $D$ visits the unassigned point and returns to $D$.)

\section{Acknowledgments}

We would like to express our gratitude to the anonymous referees for their constructive comments on the earlier version of this work. 


\section{References}

Barreto, S. S. (2004). Análise e Modelização de Problemas de localização- distribuição, Analysis and modelling of location-routing problems. Ph.D. Thesis, University of Aveiro, Aveiro, Portugal.

Barreto, S., Ferriera, C., Paixao, J., \& Santos, B. S. (2007). Using clustering analysis in a capacitated location-routing problem. European Journal of Operational Research, 179, 968-977.

Clarke, G. \& Wrights, J. W. (1964). Scheduling of vehicles form a central depot to a number of delivery points. Operations Research, 12, 568-581.

Duhamel, C., Lacomme,P., Prins, C., \& Prodhon, C. (2010). A GRASP $\times$ ELS approach for the capacitated location-routing problem. Computers \& Operations Research, 37(11), 1912-1923.

Eilon, S., Watson-Gandy, C. D. T. \& Christofides, N. (1971). Distribution management: mathematical modeling and practical analysis. New York, Hafner publishing company.

Hansen. P. \& Mladenovic, M. (2003). A tutorial on variable neighborhood serach. Les Cahiers $d u$ GERAD G-2003-46, Montreal, Canada.

Hassan-Pour, H. A., Mosadegh-Khan, M., \& Tavakkoli-Moghaddam, R. (2009). Solving a multiobjective multi-depot stochastic location-routing problem by a hybrid simulated annealing algorithm. Proc. IMechE, Part B., 223, 1045-1054.

Laporte, G. (1988). Location Routing Problems, In: Golden, B.L. and Assad, A.A. (eds), Vehicle Routing: Methods and Studies, 163-197, North-Holland, Amsterdam.

Laporte G., N., Y., \& Taillefer, S. (1988). Solving a family of multi-depot vehicle routing and location-routing problems. Transportation science, 22, 161-172.

Larson, R. C. \& Odoni, A. R. (1981). Urban Operations Research, Prentice-Hall, NJ

Lin, J.-R., \& Lei, H.-C. (2009). Distribution systems design with two-level routing considerations. Annals of Operations Research, 172 (1), 329-347.

Marinakis, Y., \& Marinaki, M. (2008). A Bilevel Genetic Algorithm for a real life location routing problem. International Journal of Logistics Research and Applications, 11 (1), 49-65.

Min, H., Jayaraman, V. \& Srivastava, R. (1998). Combined Location-Routing Problems: A Research Directions Synthesis and Future. European Journal of Operational Research, 108, 1-15.

Mladenovic, M. \& P. Hansen. P. (1997). Variable neighborhood search. Computers and Operations Research, 24,1097-1100.

Nagy, G., \& Salhi, S. (2007). Location-routing: Issues, models and methods, European Journal of Operational Research, 177, 649-672.

Prins, C., Prodhon, C., Ruiz, A., Soriano, P., \& Wolfler Calvo, R. (2007). Solving the capacitated location-routing problem by a cooperative Lagrangean relaxation granular tabu search heuristic. Transportation Science, 41(4), 470-483.

Prins, C., Prodhon, C., \& Wolfler Calvo, R. (2006). Solving the capacitated location routing problem by a GRASP complemented by a learning process and a path relinking. 4OR, 4(3), 221-238.

Schwardt, M., \& Fischer, K. (2009). Combined location-routing problems-a neural network approach, Annals of Operations Research, 167 (1), 253-269.

Simchi-Levi, D., Kaminsky, P., \& Simchi-Levi, E. (2003). Designing and Managing the Supply Chain: Concepts, Strategies, and Case Studies. Irwin, Boston: McGraw-Hill.

Talbi, E. G. (2009). Metaheuristics: From Design to Implementation. Hoboken, New Jersey. John Wiley \& Sons, Inc.

Tuzun, D., \& Bruke, L. I. (1999). A two-phase tabu search approach to the location routing problem. European Journal of Operational Research, 116, 87-99.

Yu, V. F., Lin, S-W., Lee, W., \& Ting, C-J. (2010). A simulated annealing heuristic for the capacitated location-routing problem. Computers \& Industrial Engineering, 58, 288-299. 